\title{
The Forensic APpliCATIOn OF MiCROSCOPY AND MiCROANALYSIS
}

\author{
M. Martinez, MSFS* \\ *Bexar County Criminal Investigation Laboratory, 7337 Louis Pasteur San Antonio, \\ Texas
}

In the summer of 1994, Americans sat at the edge of their seats as they watched a white Ford Bronco being followed by dozens of law enforcement vehicles on a stretch California highway. This single memorable event in history ultimately led to what many call the "Trial of the Century". As the world watched the judicial system at work from the comforts of their homes, Forensic Science was introduced as a pivotal part of the investigation. Since then the study and application of the many disciplines that encompass the Forensic Sciences, including Forensic Microscopy, has been popularized by Hollywood and educational television.

Microanalysis by microscopic methods can be simply defined as the examination, interpretation and identification of minute elements. With regard to Forensic Science, microanalysis refers to the identification of trace amounts of evidence recovered from a crime scene in order to establish a source or provide points of comparison. Forensic Scientists have many instruments in their arsenal to establish a connection of physical evidence, and perhaps one of the oldest and most underutilized instruments in the modern laboratory is the polarized light microscope or PLM. In the hands of a trained forensic microscopist, the PLM can quickly identify a variety of trace evidence such as hairs, fibers, glass, soil, explosives, and a myriad of organic and inorganic substances. An overview of forensic microanalysis dealing with trace evidence and the forensic techniques of microcrystallization used to identify explosives and explosive residues, drugs and other organic and inorganic elements will be discussed, as well as dispersion staining as a technique used to screen and identify transparent trace evidence as being different or identical with a high degree of confidence. In addition, many have questioned what role the microscope plays in the forensic community. As newer and more automated instruments become available, there is ongoing controversy in the forensic community surrounding presumptive versus confirmatory results and the possible subjectivity in interpretation that the microscope yields. The legal admissibility and testimony in court proceedings of scientific conclusions will also be addressed.

To assist forensic microscopists worldwide, the International Association for Microanalysis (IAMA) was established in 1999 with the goal of providing educational resources and support to the forensic community to enhance the quality of expertise in the field of microscopy. IAMA has sought to inform and educate scientists trained in advanced forensic microscopic methodologies in current forensic applications, research, and quality assurance. The organizational structure and membership information regarding IAMA will also be discussed. 
The images below represent the microcrystallization of two (2) explosive compounds using Polarized Light Microscopy (PLM) under full crossed polarization:

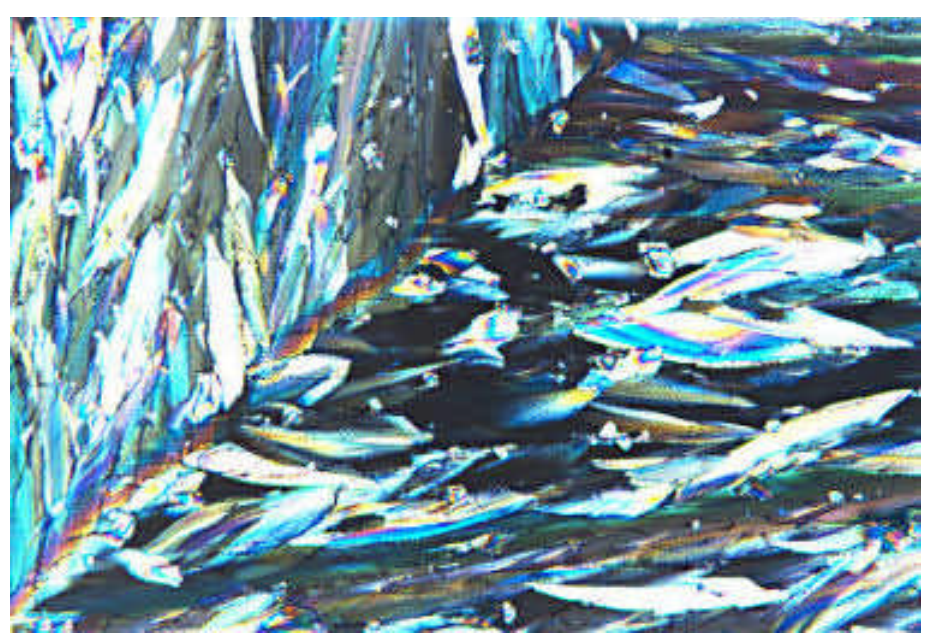

FIG. 1. 1.3.5-Trinitrotoulene

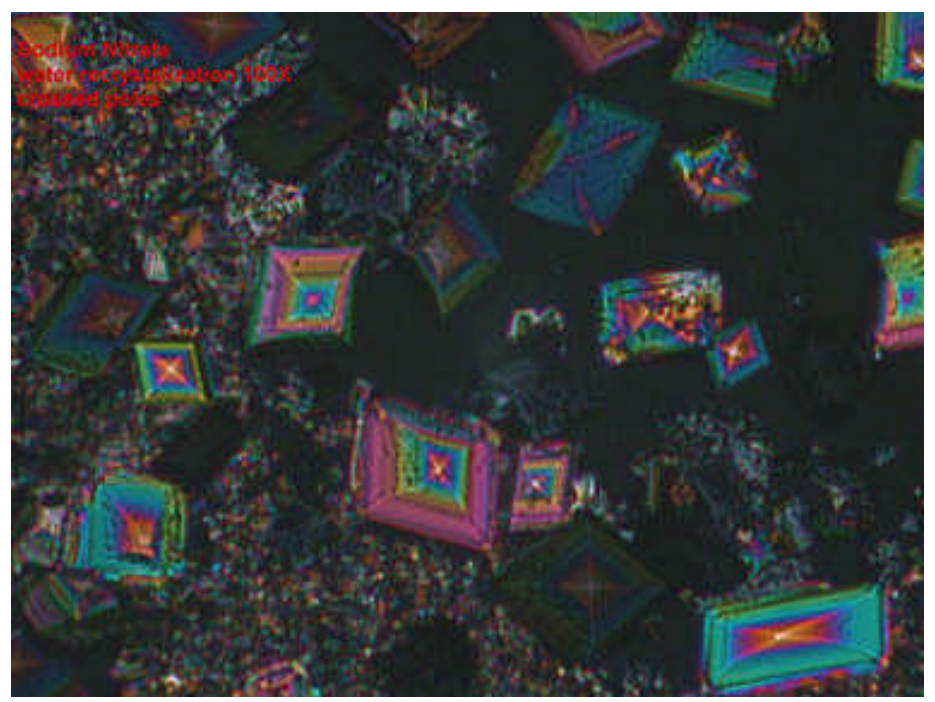

FIG. 2. Sodium Nitrate in $\mathrm{H}_{2} \mathrm{O}$ 